\title{
PRODUTIVIDADE DE CULTIVARES DE UVAS PARA SUCO SOBRE DIFERENTES PORTA-ENXERTOS IAC EM MOCOCA-SP ${ }^{1}$
}

\author{
MAURILO MONTEIRO TERRA ${ }^{2,3}$, CELSO VALDEVINO POMMER ${ }^{2,3}$, ERASMO JOSÉ PAIOLI PIRES ${ }^{2,3}$, \\ IVAN JOSÉ ANTUNES RIBEIRO ${ }^{2}$, PAULO BOLLER GALLO ${ }^{2,}$, ILENE RIBEIRO DA SILVA PASSOS ${ }^{2}$
}

\begin{abstract}
RESUMO - Estimou-se o potencial de produção de uvas das cultivares copa Concord, Isabel e Seibel 2, recomendadas para produção de suco, enxertadas sobre os porta-enxertos IAC 313 'Tropical', 'IAC 571-6', IAC 572 'Jales', e IAC 766 'Campinas', num experimento conduzido, durante seis anos, na Estação Experimental de Mococa $\left(21^{\circ} 28^{\prime}\right.$ 'S, $47^{\circ} 01^{\prime}$ W) do Instituto Agronômico de Campinas. Para a variedade Concord, as maiores produções médias foram obtidas quando se utilizaram os porta-enxertos 'IAC 313', 'IAC 571-6' e 'IAC 572 ', totalizando 3,86kg/planta, 3,81 kg/planta e 3,65kg/planta, respectivamente; para 'Isabel', quando se empregaram os porta-enxertos 'IAC 572', 'IAC 571-6' e 'IAC 313', somando 3,85kg/planta, 3,63kg/planta e 3,26kg/planta; para o cv. Seibel 2, quando se fez uso dos porta-enxertos 'IAC 313', 'IAC 572' ' 'IAC 571-6', auferiram-se 2,61kg/planta, 2,40kg/planta e 2,10kg/planta; por outro lado, para as três variedades, o porta-enxerto de pior performance foi o 'IAC 766'. Quanto ao vigor, representado pela massa dos ramos podados ao longo dos anos, os melhores porta-enxertos para 'Concord' foram 'IAC 572' e 'IAC 313'; para 'Isabel': 'IAC 766' e 'IAC 313'; e para 'Seibel 2': 'IAC 766' e 'IAC 572'.
\end{abstract}

Termos de indexação: suco de uva, cultivares, porta-enxertos, Vitis sp.

\section{YIELD OF JUICE GRAPE CULTIVARS GRAFTED ON SOME IAC ROOTSTOCKS}

\begin{abstract}
The yield potential of juice grape cultivars, namely Concord, Isabel and Seibel 2, grafted on 'IAC 313', 'IAC 571-6', 'IAC 572' and 'IAC 766' rootstocks was studied in an experiment conducted for six years at Mococa Experimental Station (21 28'S, $47^{\circ} 01$ 'W). Regarding Concord cultivar, the greatest mean yields were achieved when grafted on 'IAC 313', 'IAC 571-6' and 'IAC 572 'rootstocks, respectively $3.86 \mathrm{~kg} / \mathrm{plant}, 3.81 \mathrm{~kg} / \mathrm{plant}$ and $3.65 \mathrm{~kg} / \mathrm{plant}$. 'Isabel' gave the largest grape productions when grafted on 'IAC 572' (3.85kg/plant), 'IAC 571-6' (3.63kg/plant) and 'IAC 313' (3.26kg/plant). The best grape yields of 'Seibel-2' were obtained when it was grafted on 'IAC 313', 'IAC 572' and 'IAC 571-6', being respectively $2.61 \mathrm{~kg} / \mathrm{plant}, 2.40 \mathrm{~kg} / \mathrm{plant}$ and $2.10 \mathrm{~kg} / \mathrm{plant}$. Regardless the variety used as scion, the rootstock with the worst performance in grape production was 'IAC 766'. Vigor, measured through the weight of pruned stems along the period of the experiment, was higher when 'IAC 572' and 'IAC 313' were used for 'Concord', 'IAC 766' and 'IAC 313' for 'Isabel' and 'IAC 766' and 'IAC 572' for 'Seibel-2'.
\end{abstract}

Key words: grape juice, cultivars, rootstocks, Vitis spp

\section{INTRODUÇÃO}

O suco de uva produzido no Brasil é obtido a partir de cultivares americanas ou híbridas. No Rio Grande do Sul, principal produtor, as cultivares mais utilizadas são 'Concord' e 'Isabel'. No Estado de São Paulo, existe a possibilidade de se cultivarem uvas destinadas à fabricação de suco, e as cultivares com melhor potencial para as condições edafoclimáticas paulistas são 'Concord', 'Isabel' e 'Seibel 2'. Apesar disso, Rigitano (1976) indicava que a pequena produção paulista baseava-se em matériaprima inadequada, embora o consumo tendesse a aumentar de ano para ano.

A competitividade internacional é elevada, pois o suco brasileiro chega na costa leste americana a custos mais baixos do que aquele produzido no costa oeste dos EUA (Barber, 1997), mesmo com as taxas aplicadas.

É preciso considerar, também, a importância crescente do consumo de produtos naturais. O suco fresco de uva apresenta, por litro, 650 a $850 \mathrm{~g}$ de água, 150 a $250 \mathrm{~g}$ de açúcar redutor, 6 a 14g de ácidos orgânicos, 2,5 a 3,5g de substâncias minerais e 0,5 a $1 \mathrm{~g}$ de compostos nitrogenados (Santos et al., 1996), mantendo o bom teor de Ca, P, Fe contido na fruta. O suco comercializado internacionalmente é o concentrado a $65^{\circ}$ a $68^{\circ}$ Brix, surgindo, com expressão, os chamados sucos orgânicos produzidos a partir de uvas colhidas em vinhedos orgânicos; e virgem, isto é, livre de anidrido sulfuroso $\left(\mathrm{SO}_{2}\right)$ e outros conservantes (Vinea, 2.000).

A matéria-prima para produção do suco de uva no Brasil tem sido objeto de estudo ao longo do tempo e nas regiões produtoras. Fenochio (1973) comparou as cultivares Isabel, Concord, York Madeira, Othello, Union Village, Jefferson, Landot 234, Seibel 2, Seibel 2007 e Aspiran Bouschet, no Rio Grande do Sul. Concluiu que 'Othello' e 'Concord' são as mais convenientes para a produção de suco de uva nas condições gaúchas. Identificou que 'York Madeira', apesar do baixo rendimento em suco, presta-se bem para corte do suco de 'Isabel', melhorandoo consideravelmente, o que é relevante, considerando a abundância de 'Isabel'. Hashizume et al. (1977), em São Paulo,

1 Trabalho $\mathrm{n}^{\circ}$ 072/2000. Recebido: 18/05/2000. Aceito para publicação: 23/03/2001.

2 Pesquisador Científico do Instituto Agronômico de Campinas, Cx. Postal 28, 13001-970 Campinas, SP

3 Bolsista do $\mathrm{CNPq}$ 
considerando a grande área plantada com 'Niagara Rosada', efetuaram estudos com suco dessa cultivar, cortada com sucos de 'Seibel 2' e 'IAC 138-22', cultivares ricas em pigmentos antociânicos. O corte, com suco de qualquer dessas duas cultivares, melhorou sensivelmente o suco de 'Niagara Rosada', enquanto a aparência e o sabor foram melhores com corte de 'IAC 138-22'.

Com a disseminação da filoxera, severa praga que danifica as raízes da videira, os viticultores tiveram que fazer uso de porta-enxertos tolerantes ou resistentes à praga. Entretanto, apesar da disponibilidade razoável de bons porta-enxertos, é preciso mencionar que cada um deles tem a sua limitação, e só a experimentação regional pode determinar com regular precisão qual é o melhor (Pommer et al., 1997).

Em experimento de longa duração em Tietê e Tatuí (SP), Terra et al. (1990) pesquisaram o potencial produtivo das variedades de uvas para vinho 'IAC 138-22', 'IAC 960-9', 'IAC 116-31' e 'IAC 960-12', como produtores diretos e enxertadas sobre os porta-enxertos 'IAC 313', 'IAC 766' e '106-8 Mgt'. No conjunto dos ambientes, as maiores produções, estatisticamente superiores às demais, foram obtidas com o 'IAC 138-22' enxertado sobre o 'IAC 313' e com o 'IAC 960-9' sobre o mesmo portaenxerto. Nas condições de Tietê, o 'IAC 138-22' e'IAC 960-9' enxertados sobre o 'IAC 766' apresentaram potencial produtivo semelhante ao obtido quando sobre 'IAC 313'. O 'IAC 138-22' demonstrou maior estabilidade de produção nos ambientes estudados; o 'IAC 116-31' é de grande vigor vegetativo, medido pela quantidade de ramos podados, o que pode ter levado às suas baixas produções.

O objetivo deste trabalho, em experimento de longa duração, foi comparar o potencial de produção de uvas de três cultivares-copa indicadas para a produção de suco enxertadas sobre quatro porta-enxertos.

\section{MATERIAL E MÉTODOS}

Em Mococa-SP, na Estação Experimental do Instituto Agronômico de Campinas, situada a $21^{\circ} 28^{\prime} \mathrm{S}, 47^{\circ} 01^{\prime} \mathrm{W}, 665 \mathrm{~m}$ de altitude e clima classificado como $\mathrm{Cwa}$, em solo podzólico vermelho-escuro eutrófico, textura argilosa/muito argilosa, realizou-se um experimento de longa duração, visando ao estudo do comportamento das cultivares Concord, Isabel e Seibel 2 de uvas para suco, sobre quatro porta-enxertos IAC: 313 'Tropical', '571-6', 572 'Jales' e 766 'Campinas'.

A cultivar Concord, da espécie Vitis labrusca, foi selecionada por E. W. Bull, em Concord, Massachusetts, E.U.A. Lembra 'Isabel' e 'Niagara Rosada', pelo marcante sabor foxado, sendo, como elas, vigorosa e produtiva, apresentando satisfatória resistência ao míldio, oídio e antracnose. Cachos de tamanho médio, cilíndricos e compactos. Bagas médias, ovóides, de cor preto-azulada, polpa deliqüescente. Maturação médio-tardia.

'Isabel' é uma cultivar da espécie Vitis labrusca, originária dos Estados Unidos. Apresenta boa resistência à antracnose. Planta vigorosa, produzindo em média 1 a $2 \mathrm{~kg} / \mathrm{m}^{2}$, suporta bem a poda curta na condução em espadeira. Maturação tardia e sabor foxado. Os cachos são médios, cônicos e pouco compactos. As bagas são médias, arredondadas, de cor pretoazulada, e cobertas com abundante pruína.
A origem mais provável da cultivar Seibel 2 é o cruzamento entre Vitis lincecumii e Alicante Bouschet. De vigor e produtividade médios, é rústica a ponto de exigir poucos tratamentos fitossanitários. Cachos pequenos a médios, cônicos, às vezes alados, medianamente compactos. As bagas são pequenas, arredondadas, pretas, de sabor herbáceo. Sua acidez é elevada. Maturação tardia.

Com relação aos porta-enxertos, o 'IAC 313' originouse do cruzamento entre Golia e Vitis cinerea. Vigoroso; perfeitamente adaptado às condições climáticas paulistas; adaptase bem a diferentes tipos de solo, inclusive os que apresentam acidez elevada; suas folhas apresentam boa resistência às moléstias fúngicas; suas estacas apresentam bom índice de pegamento.

O 'IAC 571-6' originou-se do cruzamento de Vitis caribaea x Pirovano 57. Vigoroso; desenvolve-se bem tanto em solos argilosos como arenosos; folhas resistentes às principais moléstias criptogâmicas, ótimo pegamento.

O 'IAC 572' foi obtido do cruzamento entre Vitis caribaea e 101-14 Mgt. Vigoroso; vegeta bem tanto em solos argilosos como em arenosos; folhas resistentes as fitonoses; seus ramos lignificam tardiamente e dificilmente perdem as folhas; ótimo enraizamento e pegamento.

O 'IAC 766' originou-se do cruzamento entre Ripária do Traviú e Vitis caribaea. Vigoroso; apresenta perfeita adaptação às condições ambientais paulistas; suas folhas são bastante resistentes às doenças causadas por fungos; seus ramos hibernam melhor que os dos porta-enxertos 'IAC 313' e 'IAC 572'; suas estacas apresentam bom índice de pegamento.

Avaliou-se a produtividade daquelas cultivares sobre esses porta-enxertos, considerando-se a produção de uvas por seis anos (kg/planta) e o peso de ramos podados por cinco anos (grama/parcela). Completado o ciclo da cultura, com os frutos perfeitamente sazonados, efetuou-se a colheita.

O delineamento experimental usado foi o de blocos ao acaso, com 12 tratamentos e 4 repetições, totalizando 48 parcelas, cada qual constituída de 3 plantas, no espaçamento $2 \times 1 \mathrm{~m}$, conduzidas no sistema de espaldeira, com 4 fios de arame, e poda curta de inverno no cordão esporonado unilateral. Executaram-se análises de variância ano a ano, e a semelhança entre os quadrados médios residuais permitiu a análise conjunta.

\section{RESULTADOS E DISCUSSÃO}

As análises de variância revelaram diferenças significativas entre as cultivares-copa nos anos de 1984, 1987, 1988 e 1989 na produção de uvas (Tabela 1). 'Concord' foi superior a 'Seibel 2' em 1987, 1988 e 1989, sendo que, neste último ano, foi também superior a 'Isabel', nesta característica. Em 1984, 1987 e 1988, 'Isabel' mostrou maior produção que 'Seibel 2', não diferindo desta em 1989. Na média dos seis anos, 'Concord' $(3,37 \mathrm{~kg})$ e 'Isabel' $(3,40 \mathrm{~kg})$ produziram mais uva que 'Seibel 2' $(2,25 \mathrm{~kg})$, sem considerar o porta-enxerto utilizado.

Quanto aos porta-enxertos, a Tabela 1 mostra que eles induziram produções diferenciadas em 1985, 1986, 1987 e 1989. Em 1985, 'IAC 766' acarretou a menor produção de uvas, comparado com os outros três porta-enxertos. Em 1986, este porta-enxerto foi suplantado por 'IAC 571-6' e 'IAC 572', mas 
TABELA 1 - Produções (kg/planta) obtidas na E.E. de Mococa, em seis anos, das cultivares-copa utilizadas e do conjunto de cultivares-copa sobre cada um dos porta-enxertos.

\begin{tabular}{|c|c|c|c|c|c|c|c|}
\hline & \multicolumn{6}{|c|}{ A no } & \multirow{2}{*}{$\begin{array}{c}\text { M édia dos } \\
\text { anos }\end{array}$} \\
\hline & 1984 & 1985 & 1986 & 1987 & 1988 & 1989 & \\
\hline \multicolumn{8}{|l|}{ C u ltivar (C) } \\
\hline Concord & $3,91 \mathrm{ab}$ & 2,26 & 3,27 & $3,28 \mathrm{a}$ & $3,64 \mathrm{a}$ & $3,85 \mathrm{a}$ & $3,37 \mathrm{a}$ \\
\hline Is a b e 1 & $4,26 \mathrm{a}$ & 2,06 & 3,63 & $3,95 \mathrm{a}$ & $3,41 \mathrm{a}$ & $3,08 \mathrm{~b}$ & $3,40 \mathrm{a}$ \\
\hline S e ib e 12 & $2,98 \mathrm{~b}$ & 1,82 & 3,10 & $0,71 \mathrm{~b}$ & $2,38 \mathrm{~b}$ & $2,53 \mathrm{~b}$ & $2,25 \mathrm{~b}$ \\
\hline \multicolumn{8}{|c|}{ Porta-enxerto(P) } \\
\hline IA C $571-6$ & 3,85 & $2,43 \mathrm{a}$ & $3,87 \mathrm{a}$ & $2,89 \mathrm{a}$ & 3,11 & $2,92 \mathrm{ab}$ & $3,18 \mathrm{a}$ \\
\hline IA C 766 & 2,89 & $1,13 \mathrm{~b}$ & $2,58 \mathrm{~b}$ & $1,89 \mathrm{~b}$ & 2,73 & $2,64 \mathrm{~b}$ & $2,31 \mathrm{~b}$ \\
\hline IA C 313 & 4,08 & $2,29 \mathrm{a}$ & $3,38 \mathrm{ab}$ & $2,54 \mathrm{ab}$ & 3,43 & $3,74 \mathrm{a}$ & $3,24 \mathrm{a}$ \\
\hline IA C 572 & 4,06 & $2,34 \mathrm{a}$ & $3,52 \mathrm{a}$ & $3,26 \mathrm{a}$ & 3,30 & $3,32 \mathrm{ab}$ & $3,30 \mathrm{a}$ \\
\hline $\mathrm{F}($ int. $\mathrm{C} \times \mathrm{P})$ & $\mathrm{ns}$ & $\mathrm{ns}$ & $*$ & $\mathrm{n} \mathrm{s}$ & $\mathrm{n} \mathrm{s}$ & $\mathrm{ns}$ & \\
\hline $\mathrm{DM} \mathrm{S}(\mathrm{C})$ & 1,20 & 0,56 & 0,64 & 0,88 & 0,81 & 0,67 & 0,37 \\
\hline D M S (P) & 1,52 & 0,71 & 0,81 & 1,11 & 1,03 & 0,86 & 0,35 \\
\hline C. V. . (\%) & 37,0 & 31,3 & 22,0 & 36,7 & 29,8 & 24,5 & 25,8 \\
\hline
\end{tabular}

TABELA 2 - Peso (g/parcela) dos ramos podados das cultivares-copa utilizadas e peso dos ramos do conjunto das cultivares-copa sobre cada um dos porta-enxertos, em cinco anos, na E.E. de Mococa.

$$
\text { A no } \quad \text { M é dia }
$$

dos a nos

$1984 \quad 1985 \quad 1986 \quad 1987 \quad 1988$

\begin{tabular}{|c|c|c|c|c|c|c|}
\hline \multicolumn{7}{|l|}{ C ultivar (C) } \\
\hline Con cord & 643 & 1.020 & $885^{a}$ & $1.317 \mathrm{a}$ & $977 \mathrm{a}$ & $968 \mathrm{a}$ \\
\hline Is a b e 1 & 682 & 949 & $923^{a}$ & $1.242 \mathrm{a}$ & $878 \mathrm{a}$ & $832 \mathrm{~b}$ \\
\hline S e ib e 12 & 462 & 723 & $552 \mathrm{~b}$ & $655 \mathrm{~b}$ & $361 \mathrm{~b}$ & $487 \mathrm{c}$ \\
\hline \multicolumn{7}{|c|}{ Porta-enxerto (P) } \\
\hline IA C $571-6$ & 487 & 700 & $608 \mathrm{~b}$ & $722 \mathrm{~b}$ & $624 \mathrm{~b}$ & $630 \mathrm{~b}$ \\
\hline IA C 766 & 626 & 906 & $974^{\text {a }}$ & $1.242 \mathrm{a}$ & $883 \mathrm{a}$ & $926 \mathrm{a}$ \\
\hline IA C 313 & 617 & 885 & $775 \mathrm{ab}$ & $1.272 \mathrm{a}$ & $785 \mathrm{ab}$ & $867 \mathrm{a}$ \\
\hline IA C 572 & 653 & 1.099 & $790 \mathrm{ab}$ & $1.049 \mathrm{ab}$ & $664 \mathrm{~b}$ & $847 \mathrm{a}$ \\
\hline $\mathrm{F}(\mathrm{int} \cdot \mathrm{C} \times \mathrm{P})$ & $\mathrm{ns}$ & $\mathrm{n} \mathrm{s}$ & $\mathrm{N} \mathrm{s}$ & $\mathrm{ns}$ & $\mathrm{ns}$ & \\
\hline $\mathrm{D} \mathrm{M} \mathrm{S}(\mathrm{C})$ & 156 & 347 & 225 & 282 & 155 & 121 \\
\hline $\mathrm{D} M \mathrm{~S} \quad(\mathrm{P})$ & 199 & 442 & 287 & 359 & 198 & 108 \\
\hline $\mathrm{C} \cdot \mathrm{V} \cdot(\%)$ & 30,2 & 44,5 & 33,0 & 30,3 & 24,2 & 34,4 \\
\hline
\end{tabular}

igualou-se ao 'IAC 313', fato repetido em 1987. 'IAC 313' foi superior ao 'IAC 766', em 1989, e os demais foram semelhantes entre si e ao 'IAC 766'. Considerando as produções obtidas sobre esses porta-enxertos nos seis anos, 'IAC 571-6', 'IAC 572' e 'IAC 313' superaram 'IAC 766' e foram semelhantes entre si, com produções acima de $3 \mathrm{~kg}$ de uva por planta.

O 'IAC 766', embora possa ser considerado um portaenxerto de boa plasticidade, não tem mostrado performance superior em termos de produtividade, como os outros portaenxertos IAC. Em pesquisa anterior, as cultivares de uvas apirenas IAC 460-1, IAC 536-2 e IAC 871-13 foram estudadas quanto ao seu comportamento sobre os porta-enxertos 'IAC 766' e 'Ripária do Traviú'. Analisaram-se os resultados, de seis colheitas consecutivas, de 1975 a 1980, referentes à produção por planta, ao número de cachos por planta e ao teor de sólidos solúveis. Os porta-enxertos não influenciaram os resultados 
sendo consideradas satisfatórias suas atividades com a copa (Pires et al., 1992). Pommer et al. (1997) estudaram o comportamento do clone híbrido 'A1105' enxertado nos portaenxertos 'IAC 766' e 'Kober 5BB'. Na colheita, avaliaram-se produção de uvas por planta e outras características. O comportamento do 'A1105' foi semelhante sobre os dois portaenxertos, sendo a largura das bagas a única característica influenciada diferencialmente por eles. Mesmo sendo 'Ripária do Traviú' e 'Kober 5BB' porta-enxertos europeus, percebe-se que o 'IAC 766' não conseguiu superá-los, confirmando o que agora se encontrou.

Com relação ao peso dos ramos podados (Tabela 2), ocorreram diferenças entre as cultivares-copa em 1986, 1987 e 1988, sendo que, em todos, 'Concord' e 'Isabel' produziram mais ramos, independentemente dos porta-enxertos, do que 'Seibel 2 '. Já na média dos cinco anos, 'Concord' foi superior a 'Isabel' e 'Seibel 2' e 'Isabel' superior a 'Seibel 2'.

Os porta-enxertos induziram diferenças na produção de ramos em 1986, 1987 e 1988. 'IAC 766' superou 'IAC 571-6' em 1986 , sem diferenciar dos outros dois, os quais, por sua vez, não diferiram do 'IAC 571-6'. Em 1987, 'IAC 766' e 'IAC 313' induziram maior produção de ramos do que 'IAC 571-6', o qual não diferiu do 'IAC 572'. 'IAC 766', em 1988, superou 'IAC 571-6' e 'IAC 572', não se diferenciando do 'IAC 313'. A análise conjunta revelou, para esta característica, que 'IAC 766' (926g), 'IAC 313' $(867 \mathrm{~g})$ e 'IAC 572' (847g) induziram maior peso de ramos podados do que 'IAC 571-6' (630g), na média dos cinco anos avaliados.

Os quadrados médios residuais, das análises de variância ano a ano, não superaram, entre si, diferenças maiores do que cinco vezes o maior em relação ao menor, o que permitiu a análise conjunta, cujos resultados estão na Tabela 3 .

Para produção de uvas, a interação porta-enxertos $\mathrm{x}$ ano e a interação tripla não foram significativas. Para peso de ramos, a interação copa x porta-enxerto e a tripla é que não foram significativas.

Para visualizar como cada cultivar-copa produziu uvas sobre cada porta-enxerto, foi elaborada a Tabela 4. Observa-se que 'Concord' sobre 'IAC 571-6', 'Concord' sobre 'IAC 313' e 'Isabel' sobre 'IAC 572' superaram todas as outras combinações, com exceção de 'Concord'/'IAC 572'e 'Isabel'/'ÍAC571-6'. 'Seibel 2', com produções médias de uvas abaixo de $2,7 \mathrm{~kg}$ por planta, apresentou os piores resultados enxertada sobre qualquer um dos porta-enxertos.

Terra et al. (1989) estudaram o comportamento das cultivares para vinho IAC 21-14 'Madalena' e IAC 931-13 'Tetê', sobre os porta-enxertos 'RR 101-14', 'Kober 5BB', 'Ripária do

TABELA 3 - Quadrados médios obtidos nas análises conjuntas para produção de uvas (6 anos) e peso de ramos (5 anos), na E. E. Mococa.

\begin{tabular}{lrr}
\hline Fonte de variaço & Produça & Peso de ramos \\
\hline Cultivares-copa (C) & $41,09^{*}$ & $4.376 .980^{* *}$ \\
Porta-enxertos (P) & $15,84^{* *}$ & $1.004 .933^{* *}$ \\
Anos (A) & $16,35^{* *}$ & $1.528 .045^{* *}$ \\
C x P & $3,38^{* *}$ & $113.927 \mathrm{~ns}$ \\
C x A & $5,81^{* *}$ & $210.819^{* *}$ \\
P x A & $0,82 \mathrm{~ns}$ & $147.323 *$ \\
C x P A & $1,17 \mathrm{~ns}$ & $19.171 \mathrm{~ns}$ \\
\hline
\end{tabular}

Traviú', 'IAC 313' e 'IAC 766' em 3 safras consecutivas. A cultivar IAC 21-14 foi aproximadamente $50 \%$ mais produtiva que o 'IAC 931-13', apresentando, entretanto, menor teor de sólidos solúveis. As maiores produções médias para a cultivar IAC 21-14 foram obtidas quando se utilizaram os porta-enxertos 'IAC 766' e 'Ripária do Traviú' seguido de 'RR 101-14'e 'IAC 313'. A produção quando se empregou o porta-enxerto 'IAC 766', foi $257,1 \%$ maior do que quando se empregou 'Kober $5 \mathrm{BB}$ '. Os portaenxertos não influenciaram no teor dos sólidos solúveis para as duas cultivares estudadas.

Em estudo semelhante, pesquisaram-se a atividade e o potencial produtivo das cultivares de uvas brancas para vinho IAC 116-31 'Rainha' e 'IAC 960-12' sobre os porta-enxertos 'IAC 313', 'IAC 766', 'Ripária do Traviú', 'RR 101-14' e 'Kober 5BB'. Analisaram-se a produção de uvas ( $\mathrm{kg} /$ planta), o número de cachos por planta e o teor de sólidos solúveis por 3 safras consecutivas. A cultivar Rainha mostrou-se superior ao 'IAC 960-12' para os parâmetros estudados. O 'IAC 766' e o 'Ripária do Traviú, foram os melhores porta-enxertos, independentemente da cultivar-copa estudada. A pior performance foi apresentada pelo porta-enxerto 'Kober 5BB' (Pires et al., 1989).

Conforme mostra a Tabela 5, o peso de ramos podados, que é uma estimativa do vigor conferido pelo porta-enxerto, foi maior nas combinações 'Isabel'/'IAC 766', 'Concord'/'IAC 572', 'Concord'/'IAC 313', 'Concord'/'IAC 766' e 'Isabel'/'IAC 313'. 'Seibel 2' apresentou os menores pesos de ramos, independentemente da combinação com porta-enxerto. Aparentemente, o vigor induzido pelo 'IAC 766' foi excessivo, comprometendo a produtividade das cultivares-copa.

Para a produção de suco, na região em estudo, e pela aceitação internacional, recomenda-se, como opção mais adequada, o plantio da 'Concord' enxertada sobre o porta-enxerto 'IAC 313'.

TABELA 4 - Produção (kg/planta) das três cultivares-copa de uvas para suco em cada combinação de portaenxerto, na E.E. de Mococa.

\begin{tabular}{lccc}
\hline Porta-enxertos & \multicolumn{3}{c}{ Cultivares } \\
\cline { 2 - 4 } & Concord & Isabel & Seibel 2 \\
\hline IA C $571-6$ & $3,81^{\mathrm{a}}$ & $3,63 \mathrm{ab}$ & $2,10 \mathrm{efg}$ \\
IA C 766 & $2,16 \mathrm{efg}$ & $2,86 \mathrm{~cd}$ & $1,90 \mathrm{fg}$ \\
IA C 313 & $3,86 \mathrm{a}$ & $3,26 \mathrm{bc}$ & $2,61 \mathrm{de}$ \\
IA C 572 & $3,65 \mathrm{ab}$ & $3,85 \mathrm{a}$ & $2,40 \mathrm{def}$ \\
\hline D M S Tukey $=0,47$ & & &
\end{tabular}

TABELA 5 - Peso dos ramos (g/parcela) das três cultivares-copa de uvas para suco em cada combinação de portaenxerto, na E.E. de Mococa

\begin{tabular}{lccc}
\hline Porta-enxertos & \multicolumn{3}{c}{ Cultivares } \\
\cline { 2 - 4 } & Concord & Isabel & Seibel 2 \\
\hline IAC 571-6 & $827 \mathrm{c}$ & $764 \mathrm{~cd}$ & $299 \mathrm{f}$ \\
IAC 766 & $990 \mathrm{ab}$ & $1.124 \mathrm{a}$ & $664 \mathrm{de}$ \\
IAC 313 & $1.014 \mathrm{ab}$ & $988 \mathrm{ab}$ & $599 \mathrm{e}$ \\
IAC 572 & $1.042 \mathrm{a}$ & $868 \mathrm{bc}$ & $629 \mathrm{de}$ \\
\hline
\end{tabular}

DMS Tukey $=151$ 


\section{CONCLUSÕES}

1. Entre as cultivares estudadas, a 'Concord' e a 'Isabel' foram as que apresentaram as maiores produtividades médias ao longo dos anos.

2. Nas condições da região de Mococa, a produção de uva para suco foi afetada pela interação entre a cultivar-copa e o portaenxerto utilizado. As combinações 'Concord'/'IAC 313', 'Isabel'/ 'IAC 572', 'Concord'/'IAC 571-6', 'Concord'/'IAC 572' e 'Isabel'/ 'IAC 571-6', apresentaram maior potencial produtivo.

3. O porta-enxerto 'IAC 766', quanto ao vigor da planta, representada pela massa dos ramos podados, foi o que induziu maior vigor em 'Isabel' e 'Seibel 2', enquanto para a 'Concord' foi o 'IAC 572'.

\section{REFERÊNCIAS BIBLIOGRÁFICAS}

BARBER, H. The industrial Concord market from a Broker's viewpoint. 1997 Washington State Grape Society Annual Meeting Grandview (http://www.nysaes.cornell.edu/hort/faculty/ $\mathrm{pool} /$ herbbaber, janeiro 2.000).

FENOCCHIO, P. Competição de matéria-prima para elaboração de suco de uva. Pesquisa Agropecuária Brasileira, Brasília, v.8, n.8, p. 263-266, 1973.

HASHIZUME, T.; CARVALHO, R.; MORI, E.E.M.; SHIROSE, I.; VITALI, A.A. Processamento de suco de uva. Boletim do Instituto de Tecnologia de Alimentos, Campinas, v.52, p. 155-190, 1977.

PIRES, E. J. P.; POMMER, C. V.; TERRA, M. M.; SILVA, A. C. P.; PASSOS, I. R. S.; MARTINS, F.P.; COELHO, S. M. B. M.; RIBEIRO, I. J. A.; PEREIRA, F. M. Cultivares IAC de uvas de mesa apirenas sobre dois porta-enxertos. Pesquisa Agropecuária Brasileira, Brasília,v.27, n.3, p. 449-453, 1992.
PIRES, E.J.P.; MARTINS, F.P.; TERRA, M.M.; SILVA, A.C.P.; POMMER, C.V.; PASSOS, I.R.S.; COELHO, S.M.B.; RIBEIRO, I.J.A. Comportamento das cultivares IAC 116-31 e IAC 960-12 de uva branca para vinho sobre diferentes porta-enxertos. In: CONGRESSO BRASILEIRODE FRUTICULTURA, 10., Fortaleza, 1989. Anais... p. 457-460.

POMMER, C. V.; MARTINS, F. P.; PASSOS, I. R. S.; PIRES, E. J. P.; TERRA, M. M. Avaliação do clone híbrido A1105 de uvas brancas sem sementes sobre dois porta-enxertos. Bragantia, Campinas, v.56, n.1, p. 163-168, 1997.

POMMER, C. V.; PASSOS, I. R. S.; TERRA, M. M., PIRES, E. J. P. Variedades de videira para o Estado de São Paulo. Campinas:IAC, 1997. 59p (Boletim Técnico, 166).

RIGITANO, O. Considerações sobre o melhoramento de uvas para mesa e para suco no Estado de São Paulo. O Agronômico, Campinas, v. 27/28, p. 22-26, 1975/1976.

SANTOS, E.O.; DUARTE, H.A.; FRARE, S. Vinhos do RS: teores de potássio e sódio. Logos, Canoas. v. 8, n.1, p. 30-36, 1996 (http:/ /mozart.ulbra.tche.br/logos).

TERRA, M. M.; MARTINS, F. P.; PIRES, E. J. P.; POMMER, C. V.; PASSOS, I. R. S.; RIBEIRO, I. J. A.; COELHO, S. M. B. M.; SILVA, A. C. P. Cultivares de uvas moscatel para vinho sobre diferentes porta-enxertos. In: CONGRESSO BRASILEIRO DE FRUTICULTURA, 10., 1989, Fortaleza. Anais... p. 462-466.

TERRA, M.M.; PIRES, E.J.P.; PETINELLI, Jr., A.; POMMER, C.V.; SABINO, J.C.; PASSOS, I.R.S.; COELHO, S.M.B.M.; SILVA, A.C.P.; RIBEIRO, I..J.A. Produtividade de cultivares IAC de uvas para vinho como produtores direto e sobre diferentes portaenxertos. Bragantia, Campinas, v.9, n.2, p. 345-362, 1990.

VINEA. Sucos de uva. Janeiro 2.000 (http://www.comar.ar/vinea) 